Laboratory Investigation (2004) 84, 394. doi:10.1038/ labinvest.3700073

\section{Tension at the surface}

Beer's law dictates that absorbance is a function of optical density and the path length for passage of light through an absorbing substance. The definitive method for measuring the absorbance of liquid samples is passage of a light beam through a cuvette with precisely parallel sides, thereby keeping path length constant. For the most part, this is performed with one cuvette at a time. The advent of multiwell plate readers in the $1980 \mathrm{~s}$ permitted essentially simultaneous measurement of up to 96 samples, complete with calibration samples, control and test samples. This methodology is now used throughout the biological sciences; fluorescence instruments also can employ multiwell plates. However, with a multiwell plate the light path is through the flat bottom of the well and the curved air:liquid interface of the surface meniscus. Surface curvature is determined by surface tension. If there is variability in the surface tension of different samples, so there will be variable curvature of their air:liquid interfaces. Hence, slight variations in the precise location for passage of the light beam through the well may lead to substantial variability in path length, and hence error in computed optical density or fluorescence. In this issue, Cottingham et al (p. 523) present a rapid method for the measurement of surface tension in multiwell plates, based on the simple premise that a curved liquid meniscus constitutes a lens for magnification of transmitted images. They then elegantly use this property to investigate the surfactant properties of two peptides (A $\beta$ and $\mathrm{AChE}_{586-599}$ ) that form amyloid fibrils in vitro. While this methodology provides relative, rather than absolute, measurements of surface tension, this simple but powerful technique may be of considerable value for assessing the effect of macromolecular solutes on the properties of solvent liquids. Moreover, this report provides a cautionary note for the use of multiwell plates, and offers a simple methodology for evaluating the potential for artefactual error.

\section{Thrombosis and vaso-occlusion in the pathogenesis of glioblastoma}

Pseudopalisading necrosis and vascular endothelial proliferation are classic histopathologic features of glioblastoma multiforme (GBM), the most common primary brain tumour of adults. By definition, these features are not present in low-grade diffuse gliomas and their association with accelerated tumor growth and poor prognosis is well known. However, only recently have experimental and clinical studies begun to reveal clues concerning the mechanisms that result in pseudopalisading necrosis and accelerated growth in GBM. In this issue, the minireview by Brat and Van Meir (p. 397) provides an emerging molecular paradigm that reveals insights into the pathobiology of pseudopalisading necrosis and mechanisms of glioblastoma growth. According to this model, thrombosis and vaso-occlusion are early events that lead to local hypoxia and necrosis. Pseudopalisades of tumor cells adjacent to areas of necrosis appear to be formed by cells that are actively migrating away from a central focus of hypoxia. Hypoxia also stimulates angiogenesis in the form of striking vascular endothelial hyperplasia, which further promotes tumor growth. The limited efficacy of current therapies and aggressive biologic behaviour results in a dismal outcome for patients with GBM (mean survival about 50 weeks). Elucidating molecular mechanisms involved in the vascular pathology and hypoxia that lead to pseudopalisading necrosis and accelerated tumor growth may reveal novel therapeutic targets to attack this devastating tumor.

\section{Intracellular trafficking of glomerulopathic light chains}

Patients afflicted with plasma cells dyscrasias may have circulating immunoglobulin light chains that are directly nephrotoxic. Some light chains are glomerulopathic and may produce amyloidosis or light-chain deposition disease. Some other types of light chains produce proximal tubule damage, and still others are associated with distal nephron obstruction (ie, myeloma kidney). Understanding the molecular basis for this light chain - cellular interaction is likely to be important for different pathologic outcomes. In this issue, Teng et al (p. 440) utilize colocalization immunofluorescence and competition studies to identify a single receptor class on renal mesangial cells for light chains that produce amyloidosis or light-chain deposition disease. This receptor resides in caveolae present on the plasma membrane and its expression increases upon exposure to glomeulopathic but not tubulopathic light chains. These results indicate that downstream intracellular trafficking in mesangial cell is likely different for these types of light chains, with different pathologic outcomes. The authors also present data that a previously characterized tubular receptor complex comprised of the proteins cubulin/megalin is absent on mesangial cells, suggesting that the protein mediating light-chain binding to mesangial cells is distinct. 